\title{
Special issue based on selected IEEE ANTS 2013 papers
}

\author{
Ming Xia - Vinod Vokkarane
}

Published online: 26 August 2014

(C) Springer Science+Business Media New York 2014

The Seventh IEEE International Symposium on Advanced Networks and Telecommunication Systems (IEEE ANTS) (http://www.ieee-comsoc-ants.org/2013/) was held in Chennai, India, during December 15-18, 2013, at the SRM University. ANTS is the premier IEEE forum on networking and telecommunications topics in India and was financially sponsored by IEEE Communications Society. The distinguishing characteristic of IEEE ANTS is the promotion of an intense dialogue between academia and industry to bridge the gap between academic research, industry initiatives, and governmental policies. IEEE ANTS 2013 featured a rich industry program comprising of keynote addresses, invited talks, and panel discussions to complement a high-quality technical program of peer-reviewed papers, tutorials, and $\mathrm{PhD}$ student forum presentations bringing together experts from industry, academia, and government. The conference provided an exciting meeting ground for the confluence and exchange of ideas among participants from all over the world.

IEEE ANTS 2013 received a record 165 long paper submissions and 17 short paper submissions from over 24 countries. Each paper was peer-reviewed by the members of the technical program committee, and 59 long papers and 8 short papers were accepted for oral presentation. The acceptance rate for long papers was below $35 \%$. The high-quality technical program reflects the growing importance of this conference to academic and industry researchers in India and

Selected Papers from the Seventh International IEEE Symposium on Advanced Networks and Telecommunication Systems (IEEE ANTS 2013), Chennai, India.

\section{Xia $(\bowtie)$}

Ericsson Research, San Jose, CA, USA

e-mail: xiaming2006@gmail.com

V. Vokkarane

University of Massachusetts Lowell, Lowell, MA, USA worldwide. The program also featured five invited papers and an invited talk from renowned researchers in Germany, Belgium, Hungary, and the USA, and several keynote talks from influential executives from industry. The technical presentations were organized in two parallel tracks, one covering optical and wireline networks and the other wireless networking. The papers were arranged in 14 sessions across three days. The authors of eight of these papers, after careful review by the Guest Editors of this issue, were invited to submit fulllength versions for potential publication in this Special Issue of the Springer Photonic Network Communications (PNET) Journal. After a thorough review process, as followed with regular PNET submissions, all the invited papers have been selected for publication in this Special Issue.

In "Network Economics of Optical Transport Networks with Soft Decision Forward Error Correction (SD-FEC) Technology," Anuj Malik, Marco Sosa, Geoff Benett, Onur Turkcu, Vinayak Dangui, Steve Hand, Matthew Mitchell, and Serge Melle study the impact of Soft Decision Forward Error Correction (SD-FEC) technology upon network design and economics in a long-haul optical transport network. They conclude that the SD-FEC technology not only reduces the TCO (total cost of ownership), but also simplifies the network design.

In "Disaster-Aware Service Provisioning with Manycasting in Cloud Networks," S. Sedef Savas, Ferhat Dikbiyik, M. Farhan Habib, Massimo Tornatore, and Biswanath Mukherjee propose a novel disaster-aware service-provisioning scheme that multiplexes service over multiple paths destined to multiple servers/datacenters with manycasting. They show that the proposed scheme is able to achieve high survivability against link and node failures at no extra cost compared to the other survivable schemes.

In "Efficient IP Lookup using Hybrid Trie based Partitioning of TCAM based OpenFlow Switches," S Veeramani Noor 
and Mahammad Sk focus on reducing the search time of a key stored in the forwarding table in OpenFlow switches. They also proposes an efficient way to represent data and to reduce the index TCAM size with a searching time at complexity of $\mathrm{O}(\log \log \mathrm{n})$.

In "Routing, Wavelength Assignment and Spectrum Allocation for Reconfigurable Optical Mesh Networks having a combination of Fixed and Flexible Spectrum ROADM nodes," Nanda Venkata Gopal D, Sai Kishore Bhyri, and Gowri Shankar R present a novel routing, wavelength assignment and spectrum allocation (RWSA) algorithm to address the variable pass band of next-generation reconfigurable optical add/drop module (ROADM). Their scheme is verified on a sample NSFNET mesh network.

In "Fault Tolerance Mechanisms for Virtual Data Center Architectures," Sagar C. Joshi and Krishna M. Sivalingam propose a fault tolerance mechanism to handle server failures by virtual machine (VM) migration. The authors have shown that recovery from all the faults is possible, even at high server utilization. They also present a load balancing scheme to reduce the impact of server failures on the VDCs. Through performance investigation, they showed significant reduction of VMs affected by server failures.

In "Using OpenFlow to Provide Cut-through Switching in MobilityFirst," Adrian Lara, Byrav Ramamurthy, Kiran Nagaraja, Aravind Krishnamoorthy, and Dipankar Raychaudhuri introduce a general bypass capability within the MobilityFirst architecture for both individual and aggregate flow-level traffic control. Their experiments on the ORBIT and GENI testbeds show reduced number of messages processed by the controller and reduced number of flow rules to be pushed into the switches.

In "On Downstream Transmissions in EPON Protocol over Coax (EPoC),’ Partha Bhaumik, Saigopal Thota, Kira
Zhangli, Jim Chen, Hesham ElBakoury, Liming Fang, and Biswanath Mukherjee focus on downstream transmissions in EPoC (Ethernet Passive Optical Network Protocol over Coax). They study three framing approaches for downstream coax frames based on how sub-carriers in an orthogonal frequency division multiplexed (OFDM) symbol are modulated. The authors provide an analysis on the impact of various parameters such as modulation profile, symbol duration, and length of resource blocks.

In "POST: A Scalable Optical Data Center Network," Karthi Duraisamy, Chetan B. M, and Goutam Das propose a WDM-based data center interconnect called POST (Passive Optical Scalable Tree data center network). POST is able to interconnect thousands of servers and provides lowhop count paths among servers. The authors also present two switching components for efficient routing in a POST network. Their simulation results prove that POST provides a low average delay compared to traditional data center networks with good scalability.

\section{ANTS 2013 General Co-Chairs}

Krishna Sivalingam, IIT Madras, India, skrishnam@iitm.ac.in

T. Rama Rao, SRM University, Chennai, India, ramarao@email.com

\section{General Vice-Chair}

Byrav Ramamurthy, University of Nebraska Lincoln, USA, byrav@cse.unl.edu

\section{TPC Co-Chairs}

Vinod Vokkarane, Univ. of Massachusetts Lowell, USA

Goutam Das, IIT Kharagpur

Ming Xia, Ericsson, USA 